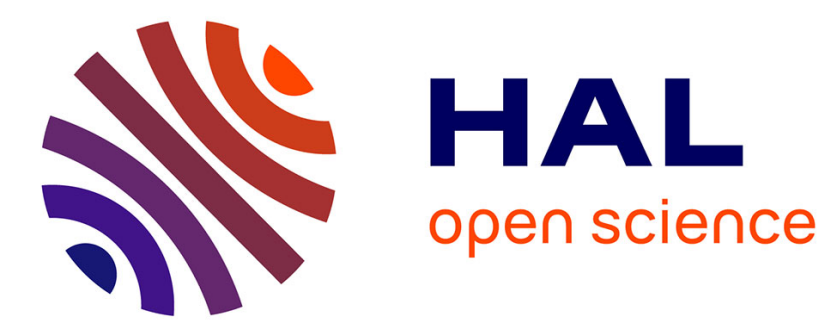

\title{
Wave-vector analysis of plasmon-assisted distributed nonlinear photoluminescence along Au nanowires
}

Deepak K Sharma, Adrian Agreda, Julien Barthes, Gérard Colas Des Francs, G V Pavan Kumar, Alexandre Bouhelier

\section{- To cite this version:}

Deepak K Sharma, Adrian Agreda, Julien Barthes, Gérard Colas Des Francs, G V Pavan Kumar, et al.. Wave-vector analysis of plasmon-assisted distributed nonlinear photoluminescence along Au nanowires. Physical Review B, 2020, 102, 10.1103/physrevb.102.115414 . hal-03025461

\section{HAL Id: hal-03025461 https://hal.science/hal-03025461}

Submitted on 26 Nov 2020

HAL is a multi-disciplinary open access archive for the deposit and dissemination of scientific research documents, whether they are published or not. The documents may come from teaching and research institutions in France or abroad, or from public or private research centers.
L'archive ouverte pluridisciplinaire HAL, est destinée au dépôt et à la diffusion de documents scientifiques de niveau recherche, publiés ou non, émanant des établissements d'enseignement et de recherche français ou étrangers, des laboratoires publics ou privés. 


\title{
Wave-vector analysis of plasmon-assisted distributed nonlinear photoluminescence along Au nanowires
}

\author{
Deepak K. Sharma $\odot,{ }^{1,2, *}$ Adrian Agreda, ${ }^{1}$ Julien Barthes $\odot,{ }^{1}$ Gérard Colas des Francs $\odot,{ }^{1}$ G. V. Pavan $\operatorname{Kumar} \odot{ }^{2}$ \\ and Alexandre Bouhelier (1) ${ }^{1, \dagger}$ \\ ${ }^{1}$ Laboratoire Interdisciplinaire Carnot de Bourgogne, UMR 6303 CNRS, Université de Bourgogne Franche-Comté, \\ 9 avenue Alain Savary, 21000 Dijon, France \\ ${ }^{2}$ Department of Physics, Indian Institute of Science Education and Research, Pune 411008, India
}

(Received 24 June 2020; revised 24 August 2020; accepted 26 August 2020; published 14 September 2020)

\begin{abstract}
We report a quantitative analysis of the wave-vector diagram emitted by nonlinear photoluminescence generated by a tightly focused pulsed laser beam and distributed along Au nanowire via the mediation of surface plasmon polaritons. The nonlinear photoluminescence is locally excited at key locations along the nanowire in order to understand the different contributions constituting the emission pattern measured in a conjugate Fourier plane of the microscope. Polarization-resolved measurements reveal that the nanowire preferentially emits nonlinear photoluminescence polarized transverse to the long axis at close to the detection limit wave vectors with a small azimuthal spread in comparison to the signal polarized along the long axis. We utilize the finite-element method to simulate the observed directional scattering by using localized incoherent sources placed on the nanowire. Simulation results faithfully mimic the directional emission of the nonlinear signal emitted by the different portions of the nanowire.
\end{abstract}

DOI: 10.1103/PhysRevB.102.115414

\section{INTRODUCTION}

Metal nanostructures confine electromagnetic fields in the subwavelength regime by controlling resonant modes known as surface plasmons polaritons (SPPs) [1,2]. The very large electric field present at the metal surface makes plasmonic-based devices excellent candidates to harness weak light-matter interactions [3,4] and to enhance optical nonlinear signals at the nanoscale [5,6]. Hand in hand with the electric field enhancement, a plasmonic nanostructure can direct the light according to the structural modes it supports [7-10]. For instance, placement of multiple elements in a specific spatial arrangement can provide unidirectional emission according to phase retardation produced by the whole geometry. A combination of plasmonic nanostructures placed in the form of the Yagi-Uda design $[11,12]$ and metasurfaces [13] have been utilized to provide high directivity and beaming effect from dipolar emitters.

In this context, a metal nanowire is an interesting plasmonic nanostructure that can confine, guide, and route SPPs [14-16]. Plasmonic nanowires confine surface plasmons well below the diffraction limit in the transverse dimensions and propagate the mode up to several micrometers along its main axis. The SPP may then out couple to free-space photons emitted in a defined angular direction when it scatters from the extremity of the metal waveguide [14]. For instance, plasmonic nanowires have been utilized for collecting and directing fluorescence signals emitted by nanocrystals [17] and quantum emitters [18] mainly in a direction imposed by the along the main axis.

\footnotetext{
*deepak-kumar.sharma@u-bourgogne.fr

†alexandre.bouhelier@u-bourgogne.fr
}

Another interesting property brought by the large enhancement of the electric field associated with the excitation of the surface plasmon is the ability of the metal structure to generate its own surface nonlinearities [19,20]. We recently showed that $\mathrm{Au}$ nanowires (AuNWs), and, more generally, extended two-dimensional structures, may produce a distributed nonlinear photoluminescence (NPL) when excited locally by a tightly focused ultrafast laser beam [21,22]. This nonlinear signal holds potential for developing advanced functionalities such as wavelength conversion [23] and all-optical Boolean operations [22,24]. Understanding this nonlinear response spatially transported in the geometry brings strategies on how to control it. Equally important is the knowledge of the direction the frequency-converted photons take when they scatter out of the structure and travel in free space.

With this motivation, we perform an analysis of the inplane wave-vector distribution of the NPL developing on the AuNW. We locally excite the NPL at the extremity and at the center of a AuNW in order to understand and discriminate contributions from the different sections of the nanowire. We analyze the resulting Fourier plane pattern emitted by the distributed NPL as a function of its polarization. We further use finite-element method based simulations to understand our experimental results.

\section{RESULTS AND DISCUSSION}

\section{A. Experimental procedures}

1. Fabrication of the Au nanowires

We fabricated a 3.5- $\mu \mathrm{m}$-long and 160-nm-wide polycrystalline AuNW on a glass substrate using a top-down fabrication procedure. Fabrication steps involve standard electron-beam lithography (Pioneer, Raith $\mathrm{GmbH}$ ) followed 


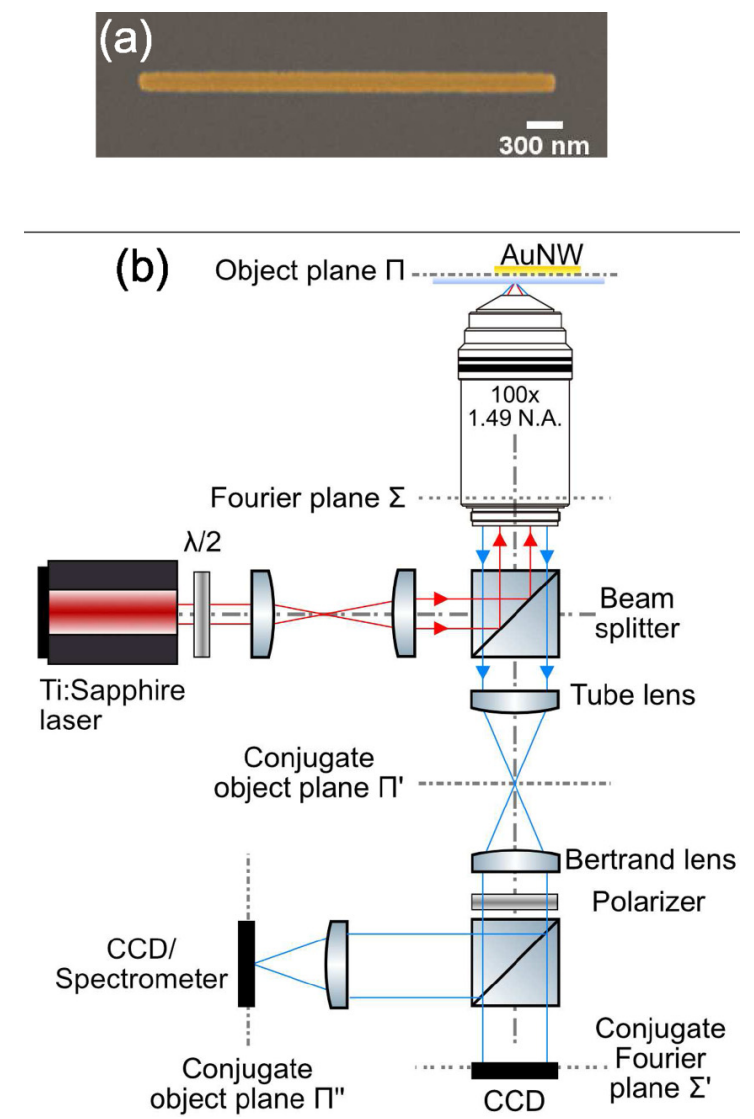

FIG. 1. (a) A scanning electron micrograph of a 3.5- $\mu \mathrm{m}$-long AuNW. (b) Schematic diagram (not to scale) of the experimental setup. The laser beam is tightly focused using a high-NA objective lens at the AuNW. Scattered optical responses into the glass substrate are collected by the same objective lens. Different combinations of relay lenses are used to capture the Fourier plane images and widefield real-plane images or spectrum from the sample. A half-wave plate is used to control input polarization, and NPL is analyzed by a linear polarizer in the output path. A set of filters is used to spectrally select the NPL response of the AuNW.

by metal depositions and a liftoff process. The subsequent metal layers consist of a 3-nm-thick adhesive layer of titanium deposited by electron-beam physical vapor deposition (MEB 400, Plassys) and a 50-nm-thick Au layer deposited by thermal evaporation. Figure 1(a) presents a scanning electron micrograph of the AuNW.

\section{Optical measurements}

The AuNW and the glass coverslip are then placed on an inverted optical microscope (Nikon, Eclipse). The experimental setup is schematically illustrated in the Fig. 1(b). The nanowire is optically excited at a wavelength of $\lambda_{0}=808 \mathrm{~nm}$ at one of its extremities by a femtosecond pulsed laser beam (Coherent, Chameleon). The beam is tightly focused through the glass by a high numerical aperture (NA) oil-immersion objective lens (Nikon, $100 \times$, NA $=1.49$ ). The same objective lens is utilized for the collection of light scattered and emitted by the nanowire. Using relay lenses placed after the exit port of the microscope, the collected light is sent to a charge-coupled device (CCD) camera (Andor, Luca) or a
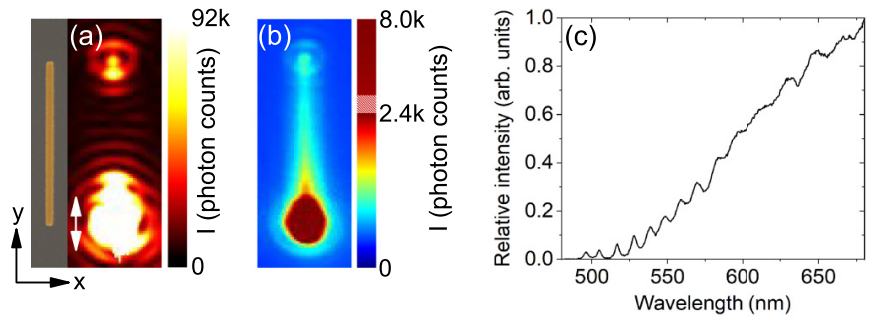

FIG. 2. (a) A wide-field real-plane image, showing surface plasmon propagation launched using a pulsed laser at $\lambda_{0}=808 \mathrm{~nm}$ focused at the nanowire's bottom end (saturated region). The white arrow indicates the polarization of the incident laser beam along the axis of the AuNW. The inset shows a scanning electron micrograph of the nanowire. (b) A false-color wide-field real-plane image showing spectrally filtered delocalized NPL from the AuNW. (c) The spectrum of NPL recorded at the excitation location. The oscillations at shorter wavelengths are due to the transmission of the filter used.

spectrometer (Andor, Shamrock 303i). We image on the CCD the back focal plane of the objective lens using relay optics in a $4-f$ configuration. This maps the projected in-plane wave-vector distribution of the light scattered or emitted by the nanowire in a conjugate Fourier plane [25]. An extra lens is used to project the wide-field real-plane image on the CCD or the spectrometer. A piezoelectric stage (MadCity Labs, Nano LP100) is utilized for the precise displacement of the nanowire within the laser beam focus. Tight focusing and geometrical discontinuity at the end of the nanowire provide enough momentum for efficient generation of SPPs in the AuNW for an incident linear polarization along the long axis of the nanowire (the $y$ axis).

SPP propagation at $\lambda_{0}$ is exemplified in the wide-field real-plane image in Fig. 2(a). The image is here taken at the laser wavelength, and the excitation appears as a saturated intensity region at the bottom end of the nanowire. The weaker luminous response at the distal end results from SPP scattering at the physical discontinuity and confirms excitation and propagation of the SPP at the pump wavelength.

Next, we increase the intensity of the laser to $\sim 7.5 \mathrm{GW} / \mathrm{m}^{2}$ to trigger a SPP-mediated nonlinear response of the nanowire. In particular, we are spectrally selecting the nonlinear photoluminescence generated and developing in the AuNW and rejecting the second-harmonic generation. NPL is spectrally filtered from the laser beam using a dichroic beam splitter (Chroma) and a set of filters (Thorlabs) placed in the collection path. The spectrally filtered wide-field real-plane image in Fig. 2(b) shows the distributed NPL over the whole structure [19,21], with the strongest response in the excitation area. NPL is an incoherent emission process [26] and consists of a wavelength continuum, as shown by the raw spectrum taken at the excitation end of the AuNW in Fig. 2(c). The spectrum in Fig. 2(c) is limited by the mentioned spectral filters set.

\section{B. NPL generation in the AuNW and its quantitative wave-vector analysis}

Next, we project the back focal plane of the objective lens onto the imaging camera to understand the wave-vector 

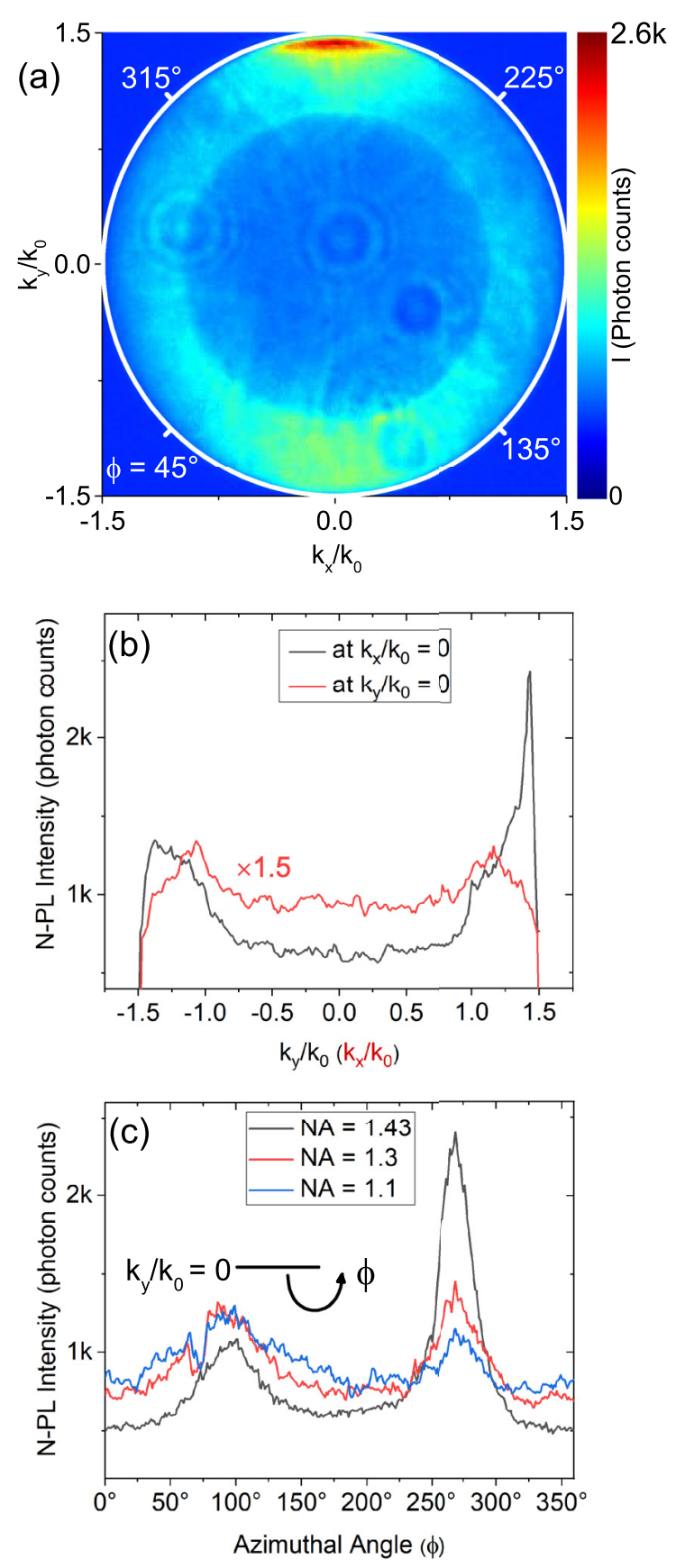

FIG. 3. (a) Fourier plane image of the delocalized NPL corresponding to the real-plane image shown in Fig. 2(b). (b) NPL intensity plots along the $k_{y} / k_{0}$ (black) and $k_{x} / k_{0}$ (red) axes of the Fourier plane image. (c) NPL intensity distribution profile along the azimuthal coordinate $\phi$ plotted at radial coordinates of 1.1, 1.3, and 1.43 .

distribution of the distributed NPL [25]. Figure 3(a) shows the Fourier plane pattern of the distributed NPL captured in the 480-680-nm wavelength range. Axes are in units of NA, where $k_{y} / k_{0}$ and $k_{x} / k_{0}$ are relative to normalized in-plane wave vectors, with $k_{0}$ being the wave vector in free space. The maximum detectable in-plane wave vector is given by the NA of the objective and sets the outer rim radius of the Fourier plane at 1.49. The inner ring is located at NA $=1.0$ and corresponds to the critical angle at the glass-air interface. The emission diagram of the NPL features an inhomogeneous distribution of the intensity, indicating that some in-plane wave vectors bear more weight than others. This is particularly the case for the $+k_{y} / k_{0}$ wave vectors located near the detection limit.

We quantitatively analyze this Fourier plane pattern by plotting radial cross sections along the $k_{y} / k_{0}$ (at $k_{x} / k_{0}=0$ ) and $k_{x} / k_{0}$ axes (at $k_{y} / k_{0}=0$ ), shown as black and red curves in Fig. 3(b), respectively. The cross sections indicate that intensity maximum is aligned along the $k_{y} / k_{0}$ axis with a peak at $k_{y} / k_{0} \sim+1.43$. As a reminder, we recall that the main axis of the AuNW is oriented along the $y$ axis. The black curve shows that the intensity in the $-k_{y} / k_{0}$ direction is smoothly distributed for $k_{y} / k_{0}>1.0$, with a small increase towards higher wave vectors in comparison to the $+k_{y} / k_{0}$ direction, where the maximum intensity clearly peaks. We hypothesize that the asymmetric distribution of intensity along the $k_{y} / k_{0}$ axis is an effect of the asymmetry of the NPL intensity distributed all along the AuNW [Fig. 2(b)], as discussed later on. The red curve shows that the intensity maxima along $k_{x} / k_{0}$ are located near the critical angle $\left( \pm k_{x} / k_{0} \sim 1.1\right)$. This feature is understood from the local excitation of the NPL by the tightly focused excitation spot. We have quantified the directionality $D=10 \log _{10} I_{\mathrm{F}} / I_{\mathrm{B}}$ along the $k_{y} / k_{0}$ axis by measuring the intensity ratio between the point with maximum intensity $I_{\mathrm{F}}$ in the upper half and the diametrically opposite point with intensity $I_{\mathrm{B}}$ in the lower half of the Fourier plane. The $D$ value is $3.15 \mathrm{~dB}$ for the intensity maximum at $k_{y} / k_{0}=+1.43$.

The Fourier plane highlights that the emitted rays not only emerge at specific $k_{y} / k_{0}$ wave vectors but also feature a limited azimuthal spread. We quantitatively evaluate the azimuthal extension by plotting the intensity as a function of the azimuthal angle $\phi$ at NA $=1.1,1.3$, and 1.43, shown in Fig. 3(c). The azimuthal plots show that the maximum NPL is angularly restricted and symmetric around the $k_{y} / k_{0}$ axis corresponding to the long axis of the AuNW. The intensity maxima are at $\phi \sim 90^{\circ}\left(-k_{y} / k_{0}\right.$ axis $)$ and $\phi \sim 270^{\circ}\left(+k_{y} / k_{0}\right.$ axis $)$, with the angular spread decreasing as NA increases in the lower half of the Fourier plane image.

In what follows, we provide an understanding of the different parts of the AuNW contributing to the complex Fourier plane pattern quantified in Fig. 3 .

\section{Position-dependent scattering of the NPL local source by the AuNW}

\section{Definition of the NPL emissive regions}

The distribution of NPL intensity across the AuNW is a result of multiple processes generating the nonlinear response [21]. Figure 4(a) schematically pictures how the NPL develops in a AuNW. Overlapping the input laser beam with a AuNW extremity results in a large electric field at the dielectric discontinuity, leading to enhanced localized NPL emission at the excitation point. This region is indicated as region I in Fig. 4(a). The excitation of the extremity launches a SPP propagating in the AuNW at the excitation wavelength $(808 \mathrm{~nm})$, as already illustrated in Fig. 2. If the intensity of the pump is large enough, the SPP triggers a nonlinear interaction as it propagates and results in the distributed NPL along the AuNW, represented as region II. This delocalized 
(a)

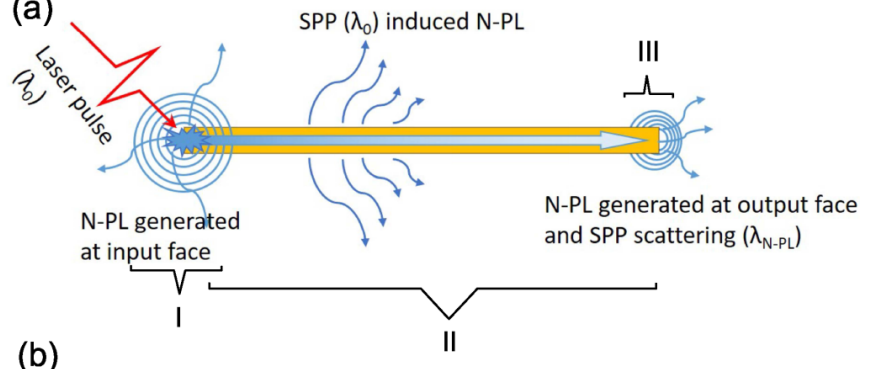

(b)

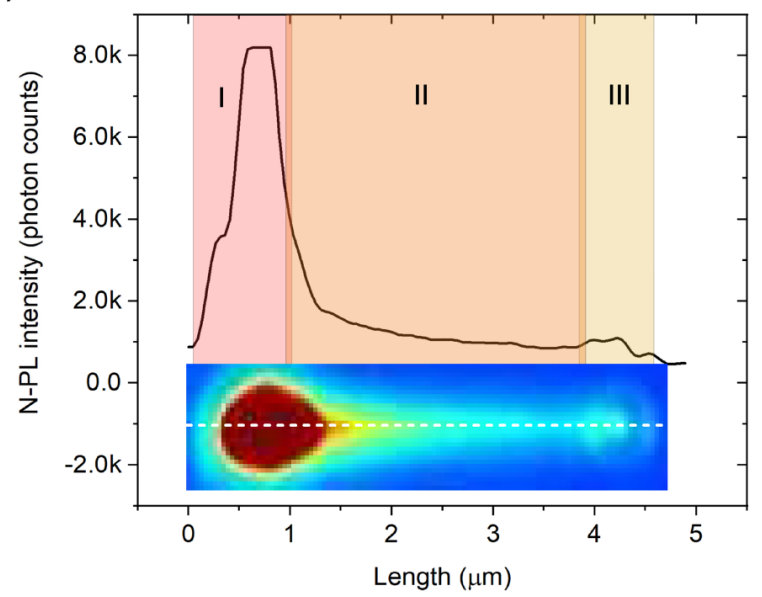

FIG. 4. (a) Schematic representation of the NPL distributed in a AuNW. (b) Intensity cross section along the AuNW indicates three different regions (I, II, and III) of the distributed NPL in the AuNW. NPL is generated at the input end of the AuNW by direct laser beam excitation (region I). A SPP traveling in the AuNW at the excitation wavelength $\left(\lambda_{0}=808 \mathrm{~nm}\right)$ generates NPL along the nanowire (region II). NPL in region III is a sum of a local NPL emission produced by the pump SPP scattering at the extremity and a continuum of SPP modes launched in region I within the NPL spectrum, traveling and out coupled by the distal end.

NPL signature is not a mode but is locally produced at the metal surface by the underlying SPP traveling at $\lambda_{0}[19,21]$. When the SPP reaches the distal end, it produces a localized NPL response, indicated as region III. Region III also contains scattering of a surface plasmon continuum. These secondary plasmons are launched at the input extremity by the strong and localized NPL produced by the laser focus. They are excited with a continuum of wavelength contained within the NPL emission spectrum (Fig. 2). These NPL SPPs out couple at $+k_{y} / k_{0}$ directions at higher wave vectors with a wide azimuthal spread [14]. This contribution is, however, not the predominant source of the NPL photons at the distal end since this set of secondary plasmons suffers from large propagation losses compared to the plasmon excited at the near-infrared pump wavelength. Considering the NPL produced at both extremities to be two local secondary sources of light, the nanowire scatters the source located in region III in the exact opposite way it scatters NPL from region I because of the mirror symmetry. However, the intensity in region III remains much weaker than that in the other two regions [see Fig. 4(b)], and we do not observe a clear symmetric $-k_{y} / k_{0}$ signature at -1.43 .

Hence, understanding the contributions from the two remaining regions, i.e., regions I and II, can provide information about the effect of different parts of the AuNW on the NPL wave-vector distribution presented in Fig. 3(a). This analysis is possible only because NPL is an incoherent process and the Fourier plane is not an interferogram.

\section{Fourier contributions from region I}

As illustrated in Fig. 4, NPL emission in region I regards the AuNW in the $+y$ axis and free space in the $-y$ axis. NPL emission in the region II perceives the presence of AuNW in both directions. To separately study the wave-vector distribution stemming from these two situations which, considering the relative weight of the nonlinear response at these positions, maximally contribute to the Fourier plane distribution in Fig. 3(a), we excite the AuNW at two different locations and for two incident polarization orientations.

First, to extract the wave-vector content of the local NPL produced by the laser in region I and scattered by the physical discontinuity, we excite one end of the AuNW with an input polarization transverse to its long axis. In this configuration, coupling to a SPP at $\lambda_{0}$ remains inefficient [27], as illustrated in the wide-field real-plane image in Fig. 5(a) taken at the excitation wavelength. Hence, NPL is generated essentially in region I and is absent from region II [Fig. 5(b)]. We observe an insignificant contribution in region III from the NPL-excited secondary SPP discussed above.

The Fourier plane image in Fig. 5(c) shows the wave-vector distribution of the NPL for this excitation configuration. We observe a strong intensity at $+k_{y} / k_{0}$ near the detection limit which strongly resembles the one measured in Fig. 3(a). This shared feature suggests that it is not the result of a SPP at $\lambda_{0}$. We claim that the reduction of the azimuthal and radial wave-vector spread is due to the quasi-one-dimensional nanowire geometry. The directivity value calculated for the intensity maximum at $k_{y} / k_{0}=+1.43$ is $D=5.6 \mathrm{~dB}$, which is in the range of directionality achieved for the optical Yagi-Uda antenna [11].

The Fourier plane in Fig. 5(c) also contains marked differences from Fig. 3(a). In particular, the intensity of the wave vectors located in the lower half of the diagram has a much larger azimuthal spread than the one measured for a polarization aligned with the nanowire. Azimuthal plots in Fig. 5(d) indicate broader wave-vector spread at NA $=1.05$ and negligible intensity at $\mathrm{NA}=1.43$ in the lower half of the Fourier plane. This can be understood from the absence of the nanowire in the $-y$ axis to the excitation spot and the absence of NPL in region II.

\section{Fourier contributions from region II}

Now, we try to understand the Fourier contribution to NPL emitted from region II of the nanowire. To remove the influence of the strong local response of the extremity, we move the laser beam to the center of the AuNW and align the incident polarization along the main axis. Here again, no SPP is launched in the AuNW because there is no momentum transfer provided by the geometry along the polarization direction. This is pictured in the wide-field image in Fig. 6(a) taken at the laser wavelength. In the laser-filtered image in Fig. 6(b), the NPL spatial extension is thus limited to the focus size with 

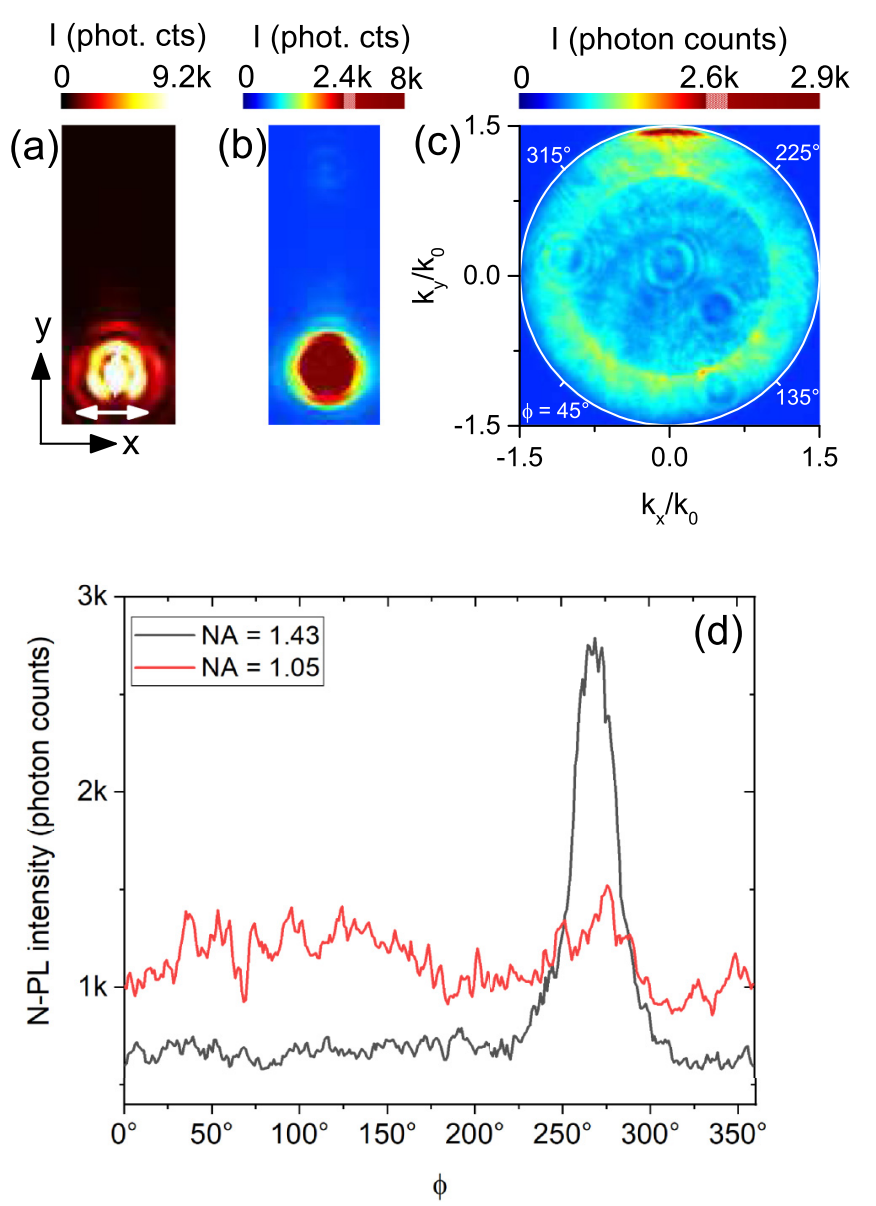

FIG. 5. (a) Wide-field real-plane image taken at $\lambda_{0}$ for an excitation polarized transverse to the nanowire. No SPP is generated for this polarization and position of the nanowire in the focus. (b) and (c) Corresponding filtered NPL wide-field real- and Fourier plane images, respectively. (d) NPL intensity distribution profile along the azimuthal coordinate $\phi$ plotted at NA $=1.05$ and NA $=1.43$. Small diffraction rings observed in the Fourier plane are from hard-toremove stains at the surface of relay lenses.

a negligible coupling of the NPL-excited secondary SPP to the nanowire. This situation mimics the effect of the nanowire on the NPL generated locally along the AuNW surface at the center of region II (Fig. 4). As expected, the presence of the nanowire on both sides of the localized NPL source renders a symmetric Fourier plane. This is pictured in Fig. 6(c), where the wave-vector intensity peaks at the detection limit on both the $+k_{y} / k_{0}$ and $-k_{y} / k_{0}$ axes. The angular azimuthal spreads are narrow on both the $+k_{y} / k_{0}$ and $-k_{y} / k_{0}$ axes, with an average $\mathrm{FWHM}=40^{\circ}$, as shown in the cross-sectional profile plotted at NA $=1.41$ in Fig. $6(d)$.

We can summarize the explanation of the Fourier plane of distributed NPL (Fig. 3) using the above observations. The maximum intensity towards $+k_{y} / k_{0}$ is due to the dominant NPL emitted in region I and redirected there by the scattering influence of the nanowire. A second contributor to this intensity maximum originates from the NPL present in region II and, to a much lesser extent, the secondary SPPs launched by the local NPL at the input and scattering in region III from the distal end. In-plane wave vectors emerging at numerical
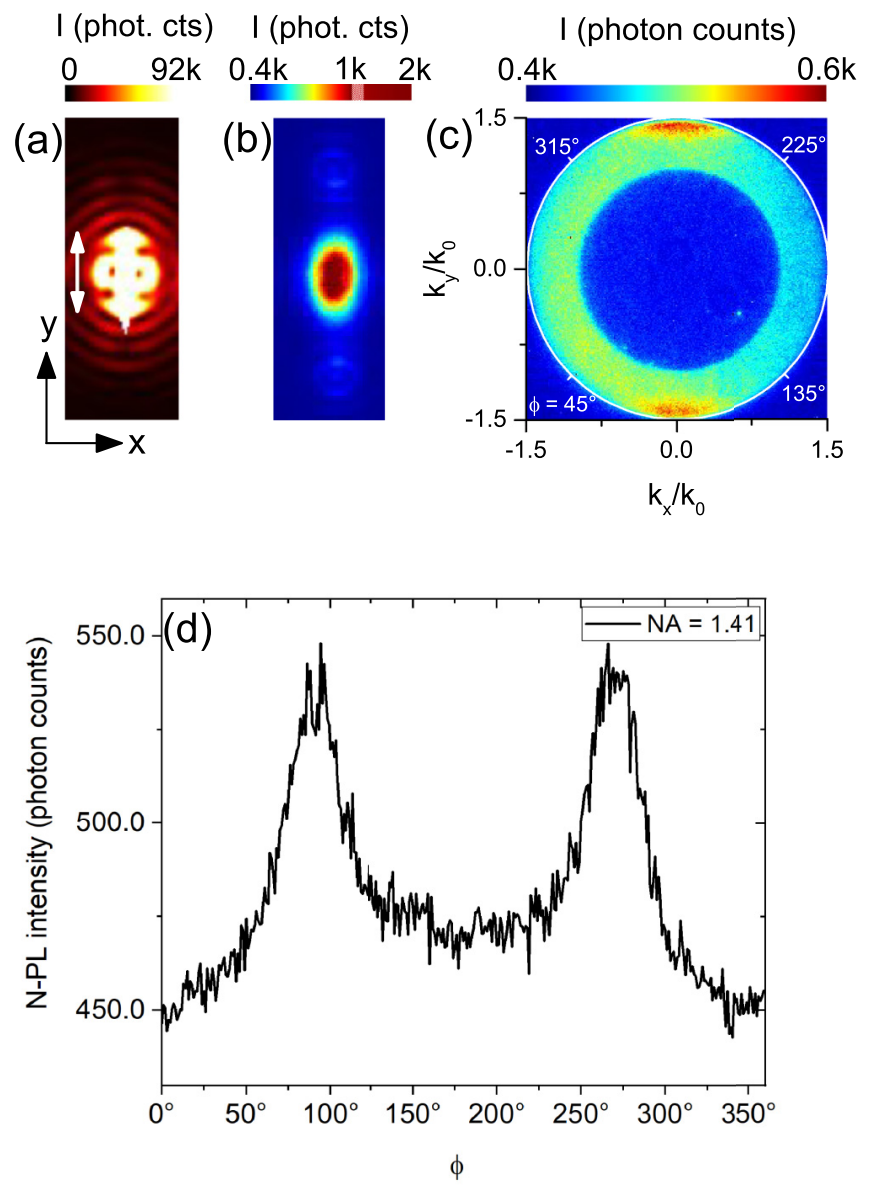

FIG. 6. (a) Wide-field real-plane image taken at $\lambda_{0}$ for an excitation located at the center of the AuNW. Polarization is along the main axis (double arrow). SPP excitation is absent. (b) and (c) Corresponding filtered NPL wide-field real-plane and Fourier plane images, respectively. (d) NPL intensity distribution profile along the azimuthal coordinate $\phi$ plotted at NA $=1.41$.

aperture values near NA $\sim 1$ in the lower half of the Fourier plane are wave vectors which are not affected by the nanowire. NPL filling the Fourier space above the critical angle in the $-k_{y} / k_{0}$ direction comes from region II complemented by the weak scattering of the localized NPL source present at the distal end (region III).

\section{Polarization-analyzed wave-vector distribution of the distributed NPL}

We complete our measurements by analyzing the polarization of the emitted NPL. We record the NPL intensity distributions in real and Fourier spaces for two polarization orientations of the emitted NPL. Polarization of the input beam remains fixed along the long axis of the AuNW, and the analyzer axis is rotated either along or transverse to the long axis of the AuNW. The excitation stays fixed at the lower extremity of the AuNW.

Figures 7(a) and 7(b) show a wide-field real-plane image and the corresponding Fourier plane image of the NPL polarized along the long axis of the AuNW, respectively. While the wide-field image does not qualitatively differ from Fig. 2, 

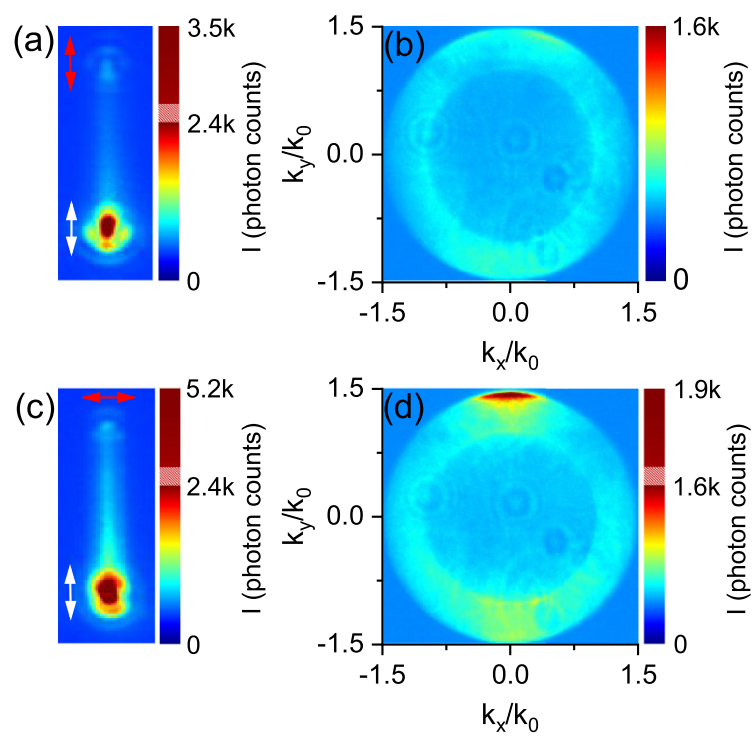

FIG. 7. Polarization-analyzed (a) and (c) wide-field real planeimages and (b) and (d) Fourier plane images. White and red arrows indicate the axis of polarization of the input laser beam and of the analyzer, respectively.

the Fourier plane is now essentially symmetric with respect to the $k_{y} / k_{0}$ and $k_{x} / k_{0}$ axes. The narrowing of the wave-vector spread in the radial and azimuthal directions at $+k_{y} / k_{0} \sim 1.43$ imparted by the nanowire is absent. We understand this from the following argumentation. We assume for the sake of the argument that the local sources positioned at either extremity and along the nanowire can be considered a random distribution of oriented dipoles. The orientation of the analyzer selects the dipole moments preferentially emitting along the nanowire. In the far field, these dipole emission diagrams features the well-known two-lobe pattern oriented perpendicularly to the dipole moment (and the axis of the nanowire). This particular orientation of the emission lobes mitigates the possible effect of the nanowire on the directionality of NPL.

Now, the analyzer axis is rotated transverse to the long axis of the AuNW to analyze the NPL polarized along this direction. The results are displayed in the wide-field realand Fourier plane images in Figs. 7(c) and 7(d). Again, the spatial distribution of the NPL closely resembles those already observed for the cross-polarization detection and for the unpolarized detection (Fig. 2). Quantitatively, the intensity of the NPL in region II is higher than in the previous polarization detection, mainly because the efficiency of the nonlinear process is helped by an interaction with the surface of the metal [21].

The Fourier plane essentially features all the important details already observed in Fig. 3. Considering again the local NPL sources at the extremities and along the nanowire to be dipoles oscillating perpendicularly to the nanowire axis, this particular orientation of the analyzer favors an emission diagram aligned with the AuNW, maximizing henceforth the directional emission and concentrating the wave-vector content to a narrow angular distribution at high $+k_{y} / k_{0}$.
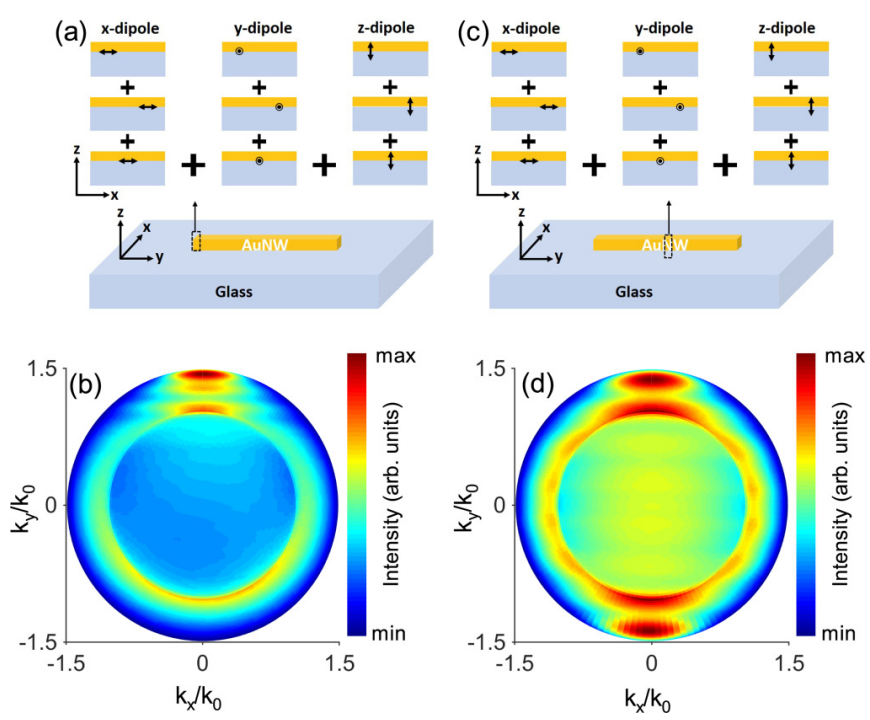

FIG. 8. (a) and (c) Schematic placement of dipoles at the end and at the center of the AuNW, respectively. Fourier plane images are simulated for a single dipole placed at positions $x=0,-40$, and +40 $\mathrm{nm}$ across the AuNW width for a wavelength range of 550-680 nm in 10-nm steps. (b) and (d) Fourier plane images representing the incoherent sum for the dipoles placed at the extremity in (a) and the center of the nanowire in (c), respectively.

\section{E. Simulation results}

We have performed a series of simulations to confirm the experimental NPL wave-vector distributions. The threedimensional finite-element method is used to calculate the near-field electric field using COMSOL MULTIPHYSICS software. The far-field wave-vector intensity distribution of the fields emitted into the substrate is calculated by an analytical treatment based on reciprocity arguments [28]. The AuNW is modeled as a 3.5- $\mu \mathrm{m}$-long, 160-nm-wide, and 50-nm-tall cuboidal structure. The cuboid is placed on a glass substrate (refractive index $=1.50$ ) and is covered with air (refractive index $=1$ ). The Au wavelength-dependent refractive index is taken from [29]. The top edges of the cuboid are rounded with a 5-nm radius to reproduce the definition of lithographically produced AuNW. A dipole source is positioned at one end of the nanowire, as schematically shown in Fig. 8(a). The extent of NPL along the width is mimicked by positioning dipoles at positions $x=0,-40$, and $+40 \mathrm{~nm}$ along the width of the AuNW at the glass-metal interface. Fourier plane patterns are simulated for dipoles with equal strength, oscillating along the $x$ (transverse to the long axis of the nanowire), $y$ (along the long axis of the nanowire), and $z$ (out of plane) axes in the NPL wavelength range (550-680 nm).

The NPL is an incoherent process, and its wave-vector distribution emitted in the substrate may be modeled as an incoherent sum of Fourier plane patterns obtained for dipoles oscillating in the $x, y$, and $z$ axes within the NPL wavelength range:

$$
\begin{aligned}
I_{\mathrm{NPL}}\left(k_{x}, k_{y}\right)= & \sum_{\lambda} a_{\lambda}\left[I_{x}\left(k_{x}, k_{y}, \lambda\right)\right. \\
& \left.+I_{y}\left(k_{x}, k_{y}, \lambda\right)+I_{z}\left(k_{x}, k_{y}, \lambda\right)\right] .
\end{aligned}
$$



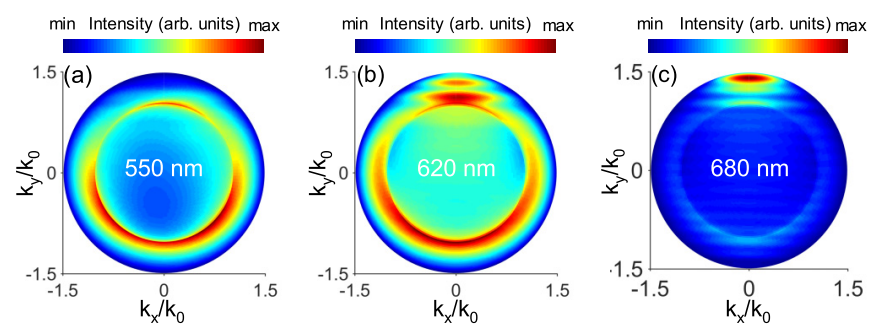

FIG. 9. (a)-(c) Calculated Fourier plane images for the incoherent dipole source placed at the end of the AuNW at single wavelengths of 550,620 , and $680 \mathrm{~nm}$, respectively.

Here $a_{\lambda}$ is a coefficient that includes the weight of the NPL at different wavelengths taken from the NPL spectrum (Fig. 2) and $I_{(x, y, z)}$ are the calculated intensity diagrams for each dipole axis. The resultant sum of $I_{\mathrm{NPL}}\left(k_{x}, k_{y}\right)$ for different positions along the $x$ axis in Fig. 8(b) resembles the experimentally observed wave-vector distribution in Fig. 5(c). The salient features observed experimentally are reproduced in the simulations, especially the distribution of intensity peaking along the $+k_{y} / k_{0}$ axis. Additional fringes not observed experimentally come from the coherent nature of dipole sources at a particular wavelength and for a particular polarization in the simulations. In contrast, NPL, by the nature of its generation, is an incoherent emission process [30].

Next, the probing dipole is moved to the center (lengthwise) of the AuNW, as shown in Fig. 8(c), and the same simulations as above are repeated to mimic the Fourier plane pattern of the NPL in Fig. 6(c). In this configuration dipoles perceive the presence of the nanowire in both the $-y$ and $+y$ directions and hence have a symmetric distribution along the $k_{y} / k_{0}$ axis [Fig. 8(d)]. The resultant incoherent sum of Fourier plane patterns in Fig. 8(d) is in qualitative agreement with the experimental distribution directions in Fig. 6(c). Hence, the simulation results qualitatively support the experimental observation that different parts of the AuNW generate NPL in-plane wave vectors differently and confirm our hypothesis.

The wave-vector distribution of NPL depends on the detection window of the spectrum [19]. For qualitative understanding of the wavelength-dependent wave-vector distribution of NPL, we have calculated Fourier plane images for the incoherent dipole source at the end of the AuNW
[Fig 8(a)] at single wavelengths of 550, 620, and $680 \mathrm{~nm}$ in Figs. 9(a)-9(c). Observed wave-vector distributions differ from each other, and Fourier plane images suggest that the intensity maximum observed in the upper half of the experimental Fourier plane image has a greater weight at higher wavelengths. This feature can be understood from material dispersion, which prevents the AuNW from operating efficiently for shorter wavelengths.

\section{CONCLUSION}

Using a quantitative analysis of Fourier plane patterns, we have demonstrated the effect of the AuNW on the directionality of NPL. Distributed NPL over the AuNW results in an intricate Fourier plane pattern, which is explained by exciting different parts of the nanowire separately. Our polarizationanalyzed study suggests that the directional signal of the distributed NPL, mapped at higher wave vectors, is maximally polarized transversely to the long axis of the nanowire. Finite-element method based simulations confirm that the directionality of the radiation pattern depends on the placement of the incoherent source at different positions on the nanowire. Our simulation results have considered a NPL source mimicked by dipoles and are in qualitative agreement with our experimental results. Hence, our study of wave-vector analysis may be equally applied to NPL generated by structures more complex than a nanowire.

\section{ACKNOWLEDGMENTS}

The work was made possible through the Indo-French Centre for the Promotion of Advanced Research, Project No. 5504-3, the APEX project funded by the Conseil Régional de Bourgogne Franche-Comté, the European Regional Development Fund (ERDF), and EUR-EIPHI Grant No. 17-EURE-0002. Device fabrication was performed in the technological platform ARCEN Carnot with the support of the Région de Bourgogne Franche-Comté and the Délégation Régionale à la Recherche et à la Technologie (DRRT). Calculations were partially performed using HPC resources from DSI-CCuB (Université de Bourgogne). G.V.P.K. acknowledges Department of science and technology, India, for a Swarnajayanti fellowship Grant No. DST/SJF/PSA-02/201718. D.K.S. thanks A. B. Vasista and D. Paul for fruitful discussions.
[1] H. Raether, Surface Plasmons on Smooth and Rough Surfaces and on Gratings (Springer, Berlin, 1988), pp. 4-39.

[2] W. L. Barnes, A. Dereux, and T. W. Ebbesen, Surface plasmon subwavelength optics, Nature (London) 424, 824 (2003).

[3] P. Anger, P. Bharadwaj, and L. Novotny, Enhancement and Quenching of Single-Molecule Fluorescence, Phys. Rev. Lett. 96, 113002 (2006).

[4] J. Langer et al., Present and future of surface-enhanced raman scattering, ACS Nano 14, 28 (2020).

[5] M. Kauranen and A. V. Zayats, Nonlinear plasmonics, Nat. Photonics 6, 737 (2012).
[6] S. Kim, J. Jin, Y.-J. Kim, I.-Y. Park, Y. Kim, and S.-W. Kim, High-harmonic generation by resonant plasmon field enhancement, Nature (London) 453, 757 (2008).

[7] C. Huang, A. Bouhelier, G. Colas des Francs, A. Bruyant, A. Guenot, E. Finot, J.-C. Weeber, and A. Dereux, Gain, detuning, and radiation patterns of nanoparticle optical antennas, Phys. Rev. B 78, 155407 (2008).

[8] T. H. Taminiau, F. D. Stefani, and N. F. van Hulst, Optical nanorod antennas modeled as cavities for dipolar emitters: Evolution of sub- and super-radiant modes, Nano Lett. 11, 1020 (2011). 
[9] D. Vercruysse, Y. Sonnefraud, N. Verellen, F. B. Fuchs, G. Di Martino, L. Lagae, V. V. Moshchalkov, S. A. Maier, and P. Van Dorpe, Unidirectional side scattering of light by a singleelement nanoantenna, Nano Lett. 13, 3843 (2013).

[10] L. Novotny and N. Van Hulst, Antennas for light, Nat. Photonics 5, 83 (2011).

[11] A. G. Curto, G. Volpe, T. H. Taminiau, M. P. Kreuzer, R. Quidant, and N. F. van Hulst, Unidirectional emission of a quantum dot coupled to a nanoantenna, Science 329, 930 (2010).

[12] T. Kosako, Y. Kadoya, and H. F. Hofmann, Directional control of light by a nano-optical Yagi-Uda antenna, Nat. Photonics 4, 312 (2010).

[13] K. Lindfors, D. Dregely, M. Lippitz, N. Engheta, M. Totzeck, and $\mathrm{H}$. Giessen, Imaging and steering unidirectional emission from nanoantenna array metasurfaces, ACS Photonics 3, 286 (2016).

[14] T. Shegai, V. D. Miljkovic, K. Bao, H. Xu, P. Nordlander, P. Johansson, and M. Käll, Unidirectional broadband light emission from supported plasmonic nanowires, Nano Lett. 11, 706 (2011).

[15] P. Geisler, E. Krauss, G. Razinskas, and B. Hecht, Transmission of plasmons through a nanowire, ACS Photonics 4, 1615 (2017).

[16] H. Wei, D. Pan, S. Zhang, Z. Li, Q. Li, N. Liu, W. Wang, and H. $\mathrm{Xu}$, Plasmon waveguiding in nanowires, Chem. Rev. 118, 2882 (2018).

[17] N. Hartmann, D. Piatkowski, R. Ciesielski, S. Mackowski, and A. Hartschuh, Radiation channels close to a plasmonic nanowire visualized by back focal plane imaging, ACS Nano 7, 10257 (2013).

[18] Q. Li, H. Wei, and H. Xu, Quantum yield of single surface plasmons generated by a quantum dot coupled with a silver nanowire, Nano Lett. 15, 8181 (2015).

[19] S. Viarbitskaya, O. Demichel, B. Cluzel, G. Colas des Francs, and A. Bouhelier, Delocalization of Nonlinear Optical Responses in Plasmonic Nanoantennas, Phys. Rev. Lett. 115, 197401 (2015).
[20] A. de Hoogh, A. Opheij, M. Wulf, N. Rotenberg, and L. Kuipers, Harmonics generation by surface plasmon polaritons on single nanowires, ACS Photonics 3, 1446 (2016).

[21] A. Agreda, D. K. Sharma, S. Viarbitskaya, R. Hernandez, B. Cluzel, O. Demichel, J.-C. Weeber, G. Colas des Francs, G. P. V. Kumar, and A. Bouhelier, Spatial distribution of the nonlinear photoluminescence in au nanowires, ACS Photonics 6, 1240 (2019).

[22] U. Kumar, S. Viarbitskaya, A. Cuche, C. Girard, S. Bolisetty, R. Mezzenga, G. Colas des Francs, A. Bouhelier, and E. Dujardin, Designing plasmonic eigenstates for optical signal transmission in planar channel devices, ACS Photonics 5, 2328 (2018).

[23] S. B. Hasan, F. Lederer, and C. Rockstuhl, Nonlinear plasmonic antennas, Mater. Today 17, 478 (2014).

[24] S. Viarbitskaya, A. Teulle, R. Marty, J. Sharma, C. Girard, A. Arbouet, and E. Dujardin, Tailoring and imaging the plasmonic local density of states in crystalline nanoprisms, Nat. Mater. 12, 426 (2013).

[25] A. B. Vasista, D. K. Sharma, and G. V. P. Kumar, Fourier plane optical microscopy and spectroscopy, in Digital Encyclopedia of Applied Physics (Wiley-VCH Verlag GmbH \& Co. KGaA, 2019).

[26] G. T. Boyd, Z. H. Yu, and Y. R. Shen, Photoinduced luminescence from the noble metals and its enhancement on roughened surfaces, Phys. Rev. B 33, 7923 (1986).

[27] M. Song, J. Dellinger, O. Demichel, M. Buret, G. Colas des Francs, D. Zhang, E. Dujardin, and A. Bouhelier, Selective excitation of surface plasmon modes propagating in $\mathrm{Ag}$ nanowires, Opt. Express 25, 9138 (2017).

[28] J. Yang, J.-P. Hugonin, and P. Lalanne, Near-to-far field transformations for radiative and guided waves, ACS Photonics 3, 395 (2016).

[29] P. B. Johnson and R.-W. Christy, Optical constants of the noble metals, Phys. Rev. B 6, 4370 (1972).

[30] V. Remesh, M. Stührenberg, L. Saemisch, N. Accanto, and N. F. van Hulst, Phase control of plasmon enhanced two-photon photoluminescence in resonant gold nanoantennas, Appl. Phys. Lett. 113, 211101 (2018). 